\title{
Non-Surgical Therapy for Peri-Implant Diseases: a Systematic Review
}

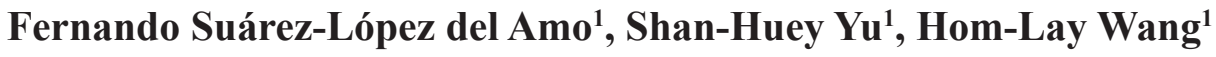 \\ ${ }^{1}$ Department of Periodontics and Oral Medicine, University of Michigan, Ann Arbor Michigan, USA.
}

\author{
Corresponding Author: \\ Hom-Lay Wang \\ Department of Periodontics and Oral Medicine \\ University of Michigan School of Dentistry \\ 1011 North University Avenue, Ann Arbor, Michigan 48109-1078 \\ USA \\ Phone: (734) 763-3383 \\ Fax: (734) 936-0374 \\ E-mail: homlay@umich.edu
}

\begin{abstract}
Objectives: The purpose of this paper was to systematically evaluate the effectiveness of non-surgical therapy for the treatment of peri-implant diseases including both, mucositis and peri-implantitis lesions.

Material and Methods: An electronic search in two different databases was performed including MEDLINE (PubMed) and EMBASE from 2011 to 2016 . Human studies reporting non-surgical treatment of peri-implant mucositis and peri-implantitis with more than 10 implants and at least 6 months follow up published in English language were evaluated. A systematic review was performed to evaluate the effectiveness of the different methods of decontamination employed in the included investigations. Risk of bias assessment was elaborated for included investigations.

Results: Twenty-five articles were identified of which 14 were further evaluated and included in the analysis. Due to significant heterogeneity in between included studies, a meta-analysis could not be performed. Instead, a systematic descriptive review was performed. Included investigations reported the used of different methods for implant decontamination, including selfperformed cleaning techniques, and professionally delivered treatment such as laser, photodynamic therapy, supra-/submucosal mechanical debridement, and air-abrasive devices. Follow-up periods ranged from 6 to 60 months.

Conclusions: Non-surgical treatment for peri-implant mucositis seems to be effective while modest and not-predictable outcomes are expected for peri-implantitis lesions. Limitations include different peri-implant diseases definitions, treatment approaches, as well as different implant designs/surfaces and defect characteristics.
\end{abstract}

Keywords: antibiotic prophylaxis; dental scaling; local anti-infective agents; peri-implantitis.

\author{
Accepted for publication: 7 September 2016 \\ To cite this article: \\ Suárez-López del Amo F, Yu SH, Wang HL. \\ Non-Surgical Therapy for Peri-Implant Diseases: a Systematic Review \\ J Oral Maxillofac Res 2016;7(3):e13 \\ URL: http://www.ejomr.org/JOMR/archives/2016/3/e13/v7n3e13.pdf \\ doi: $\underline{10.5037 / j o m r .2016 .7313}$
}




\section{INTRODUCTION}

During the last decades the definition of periimplantitis has suffered several modifications with the development in the understanding of dental implantology and its biological implications. Recently, as described by the American Academy of Periodontology [1] mucositis is defined as an inflammatory process around a dental implant without loss of supporting bone beyond biological bone remodelling. On the other hand, periimplantitis is characterized by both, inflammation of the surrounding peri-implant tissues and loss of supporting bone beyond initial biological bone remodelling. Nonetheless recent investigations have recognized at least 7 definitions of peri-implantitis based on the extension and severity of the bone loss [2]. These interpretations of the peri-implant disease certainly reflect the multifactorial nature of the entity, displaying a multitude of clinical presentations. Hence, it is reasonable to assume that further definitions will appear in upcoming years as we continue performing research in the field. Nonetheless, the complex mechanisms that influence initial bone remodelling, among other variables, will certainly ensure this to be a challenging duty.

With regard to the treatment of the peri-implant diseases, a variety of different approaches have been proposed including but not limited to: non-surgical therapy; surgical management by means of access flap debridement, lasers disinfection, implantoplasty, resective procedures, as well as regenerative approaches [3]. While most of the available evidence agrees on the effectiveness of non-surgical therapy for mucositis lesions [4], conflicting results are found when trying to identify the most effective protocol for peri-implantitis $[\underline{3}, \underline{5}]$. In this sense, a recent systematic review evaluating the effectiveness of periimplantitis treatment has shown that reconstructive procedures do not result in more optimal outcomes when compared to non-reconstructive procedures [5]. This result is in concordance with the majority of the literature available with regard to peri-implantitis treatment which presents with great variability in terms of treatment outcomes. These inconsistencies can be attributed to a variety of different factors, including but not limited to: different aetiologies and contributing factors affecting dental implants, morphology of the defects, case description/selection, implant positioning, and the influence of different implant surfaces.

Non-surgical therapy for peri-implant diseases has traditionally been considered effective for mucositis.
However, results for peri-implantitis lesions were found not to be effective [4]. Surprisingly, newer studies have challenged again this hypothesis achieving exceptional results after non-surgical decontamination of peri-implantitis lesions [] $]$.

Due to the increasing prevalence of both peri-implant mucositis and peri-implantitis, there is an urgent need to understand its aetiology and the multiples variables affecting it development and progression leading to the generation of more predictable treatment approached. Hence, the aim of the present review was to systematically evaluate the effectiveness of current (last 5 years) methodologies for the non-surgical treatment of peri-implant diseases.

\section{MATERIAL AND METHODS Protocol and registration}

The methods as well as inclusion/exclusion criteria employed for the present review were determined in advance. This protocol was registered in an international prospective register of systematic reviews 'PROSPERO' with the following registration number: CRD42016037631. The current systematic review was performed by two independent reviewers following the PRISMA guidelines for identification, screening, eligibility, and inclusion [7].

\section{Focus question}

The following focus question was developed according to the population, intervention, comparison, and outcome (PICO) study design: In patient suffering from peri-implant mucositis or peri-implantitis, what is the effectiveness of non-surgical therapy by means of different techniques and/or approaches for clinical and radiographically resolution of disease, including bleeding on probing (BOP), probing pocket depth (PPD), and radiographic bone (RB) level changes.

\section{Information sources}

The search strategy consisted in the examination of several databases as well as manual screening. The electronic search was performed in several databases, including MEDLINE (PubMed), and EMBASE databases for articles from 2011 up to April 2016 with limitation to English language. Additionally, a manual search of periodontics/implantology-related journals, including "Clinical Oral Implant Research", "Journal of Dental Research", "Journal of Clinical Periodontology", "Journal of Periodontology", "Clinical Implant Dentistry and Related Research", 
and "The International Journal of Periodontics \& Restorative Dentistry", from 2011 up to April 2016 was also performed to ensure a thorough screening process. Furthermore, references of all articles reviewed in full text were further screened.

\section{Search}

Both PubMed and EMBASE databases were screened through advances searchers. For the PubMed library, combinations of controlled terms (MeSH and EMTREE) and keywords were used whenever possible. Into the addition, other terms not indexed as MeSH and filters were applied. As such, the key terms used were: $\quad((()(()(C()(($ “non-surgical”) $)$ OR "dental prophylaxis" [MeSH Terms]) OR "dental scaling" [MeSH Terms]) OR "scaling, subgingival" [MeSH Terms]) OR "dental polishing" [MeSH Terms]) OR "diode lasers" [MeSH Terms]) OR "yag laser, erbium" [MeSH Terms]) OR "antibiotic prophylaxis" [MeSH Terms]) OR "agents, local anti infective" [MeSH Terms]) AND "periimplantitis" [MeSH Terms]) OR "peri-implant mucositis") OR "peri-implant maintenance") OR "implant infection") OR "peri-implant infection" AND ("last 5 years" [PDat] AND "Humans" [Mesh]). On the other hand, for EMBASE for following terms were employed: "non-surgical"" OR "scaling", OR "laser/exp" OR "laser" OR "subgingival curettage/exp" OR "subgingival curettage" AND ("periimplantitis/exp" OR “"periimplantitis") OR "peri-implant mucositis".

\section{Selection of studies}

Titles and abstracts derived from the search were independently screened by two reviewers (FSLA and SHY) based on the inclusion criteria. Both reviewers compared decisions and their eligibility for this review was confirmed after discussion. Full articles were obtained for all the investigations deemed eligible for inclusion in this paper and further evaluated by both reviewers. If needed, a third party was consulted when consensus could not be reached.

\section{Types of publications}

The present review included only human studies published in the English language. Letters, editorials, reviews and meta-analysis, $\mathrm{PhD}$ thesis, as well as abstracts were not evaluated.

\section{Types of studies}

The present investigation included cases series, prospective, as well randomized controlled trials (RCTs) published between 2011 and April of 2016 that reported on non-surgical treatment for periimplant mucositis and peri-implantitis. Case report, retrospective, as well as studies with less than 10 implants or less than 6 months follow up were excluded.

\section{Types of participants/population}

Individuals included in the studies should have had at least one osseointegrated screw-type dental implant that presented with signs of peri-implant mucositis or peri-implantitis and received non-surgical treatment. Nonetheless, included investigations presented with different definitions for the diseases evaluated.

\section{Inclusion and exclusion criteria}

Articles were included in this systematic review if they met the following inclusion criteria:

- Investigated non-surgical treatment outcomes for peri-implant mucositis and peri-implantitis in patients with at least one osseointegrated solid screw-type implant;

- All human prospective studies, as well as clinical trials, cohort studies, case-control, and case series studies;

- At least 10 implants;

- At least 6 months follow-up;

- Clinical and/or radiographic changes reported. Treatment outcomes reporting changes in PPDs and/or BOP and/or RB changes.

On the contrary, the following articles were excluded:

- Care reports, retrospective investigations, in vitro and animal studies;

- Less than 10 implants;

- Less than 6 months of follow-up;

- Surgical treatment for peri-implantitis;

- Human trials with missing information or unclear data.

\section{Sequential search strategy}

Initial literature search was conducted in several databases including MEDLINE (PubMed) and EMBASE from 2011 to 2016. All articles titles were screened in order to eliminate non-qualifying studies. Next, screening of abstract was performed followed by elimination of non-qualifying investigations. Finally, full text evaluation of each article was performed in order to confirm the eligibility based on the inclusion and exclusion criteria. 
References of full text evaluated investigations were also performed. In addition, a manual search in periodontics/implantology-related journals, including "Clinical Oral Implant Research", "Journal of Dental Research", "Journal of Clinical Periodontology", "Journal of Periodontology", "Clinical Implant Dentistry and Related Research", and "The International Journal of Periodontics \& Restorative Dentistry", from 2011 up to 2016, was also performed to ensure a thorough screening process.

\section{Data extraction}

Data were extracted from the included studies independently by two reviewers (FSLA and ESHY). If any disagreement occurred, a third reviewer was consulted (HLW).

\section{Assessment of methodological quality}

The quality of all selected RCTs was assessed using The Cochrane Collaboration's tool for assessing risk of bias in randomised trials [ 8$]$. Parameters evaluated included:

- Random sequence generation;

- Allocation concealment;

- Blinding of participants and personnel;

- Blinding of outcome assessment;

- Incomplete outcome data;

- Selective reporting;

- Other bias.

The potential risk of bias was categorized as "low", "unclear" or "high" depending on the quality and detailed explanation of provided information about all abovementioned parameters. All assessments were completed by a single examiner (SHY). The Newcastle-Ottawa Scale (NOS) was used to evaluate the methodological quality of nonrandomized included studies [9]. The topics evaluated were selection of study groups, comparability of patients, and outcome. Each included study received a maximum of 10 .

\section{Data analyses}

Significant heterogeneity between publications in terms of diseases definitions, study designs, patient and defect related characteristics, as well as measured outcomes, among others, prevented the quantitative synthesis of the included studies and consequently a meta-analysis could not be completed. Instead, a qualitative descriptive analysis of the reported outcomes was performed and systematically reviewed in forms of tables.

\section{RESULTS \\ Study selection}

Initial screening of electronic databases yielded a total of 2837 articles. Additionally, 21 more articles were found through manual screening. After removal of duplicated studies, a total of 2625 titles and abstract were evaluated. Overall, a total of 26 potentially relevant articles were selected after an evaluation of their titles and abstracts. Full text of these articles was obtained and thoroughly evaluated. Of these, 14 studies fulfilled the inclusion criteria and were subsequently included in the systematic review (Figure 1 and Tables 1 - 2).

\section{Excluded studies}

The reasons for exclusion are summarized in Table 3. Four articles presented with short follow up with less than 6 months [10-13], two articles were retrospective in design $[\underline{6}, \underline{14}]$, one investigation was a review study [15], three articles employed the same study population than other included investigations in the present analysis [16-18], and 1 investigation presented with unclear and incomplete data [19].

\section{Study characteristics}

The characteristics of the 14 included articles are summarized in Tables 1 and 2. The publications include several study types: 9 randomized controlled trials (RCTs) with parallel designs [20-28], 1 singlearmed cohort study [29], 4 non-controlled prospective studies and case series [30-33]. The follow-up periods of the studies ranged from 6 to 60 months. There were several different definitions regarding periimplant mucositis and peri-implantitis, being the definitions for peri-implantitis more variable than those of mucositis. In the Deppe et al. [32] study, they classified peri-implantitis into moderate and severe groups based on the severity of the bone loss.

Most articles reported the subject numbers as well as the implants evaluated in the studies, however 3 articles failed to report the numbers of the implants that were evaluated in the studies $[\underline{23}, \underline{24}, \underline{27}]$ while 1 article only presented the mean number of implants for each patient [료].

Regarding the description of the diseased sites, most studies provided information on PPD and BOP of the affected implants, however, 2 articles did not present PPD for the implants affected by

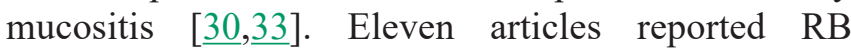
loss $[20-28, \underline{32}, \underline{33}]$. Some articles reported on 


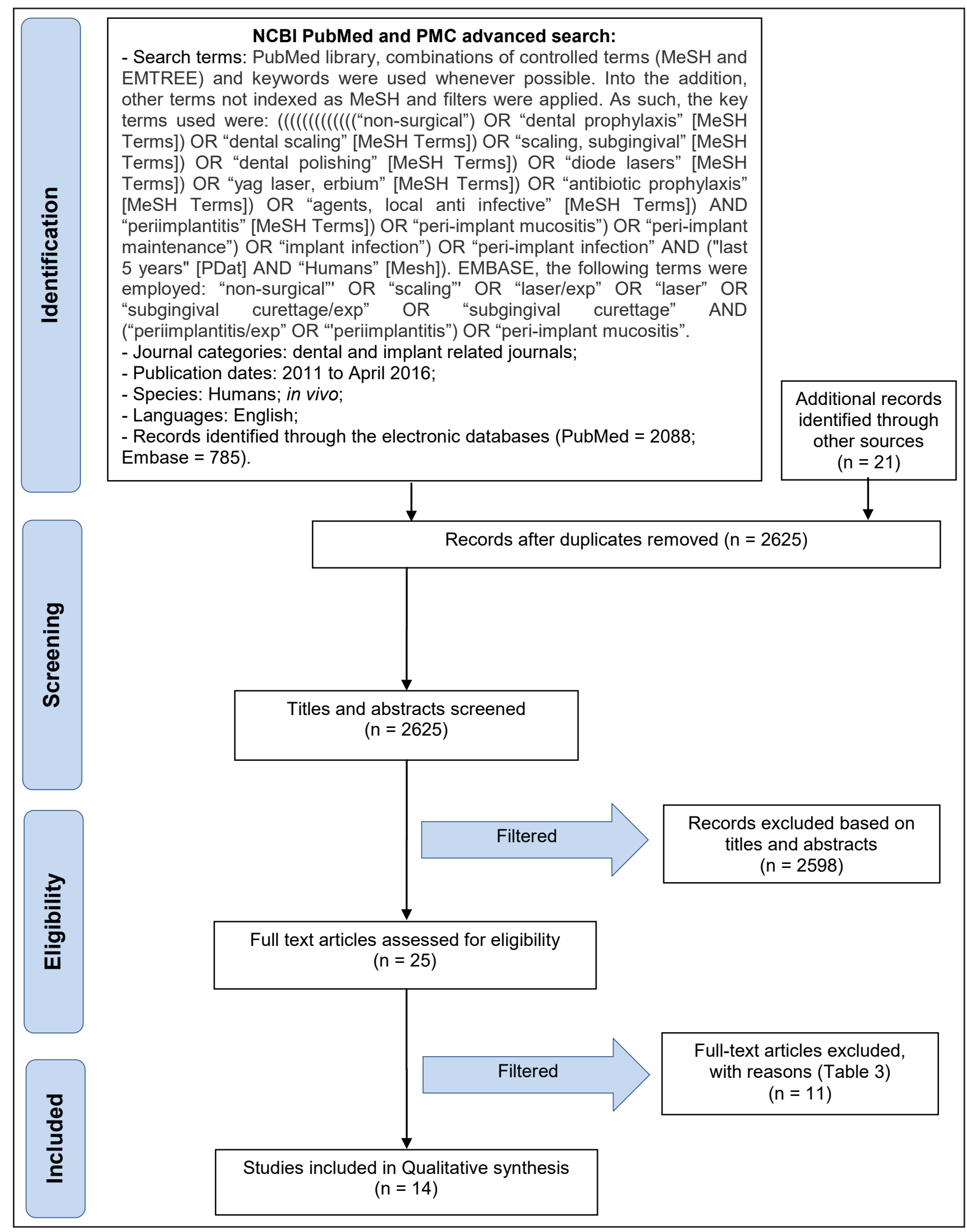

Figure 1. PRISMA flowchart of the screening process in the different databases.

the implant characteristics including the systems and the surface materials, implant functioning time and the prosthetic design $[20-24,27-29,31,33]$. One study particularly excluded implants with titanium plasma sprayed or hydroxyapatite coated implants [25]. Five articles performed microbial test $[\underline{20}, \underline{21}, \underline{23}, \underline{26}, \underline{28}]$.
Some articles reported the locations (i.e. maxilla or mandible; anterior or posterior) of the implants evaluated in the studies, however, none of the articles presented the bucco-lingual position of the implants and no articles reported the possible aetiology and/or contributory factors of peri-implant mucositis or peri-implantitis. 
Table 1A. Characteristics of the included articles

\begin{tabular}{|c|c|c|c|c|c|c|c|c|c|c|c|c|c|c|c|c|c|c|c|}
\hline \multirow[b]{2}{*}{ Study } & \multirow[b]{2}{*}{\begin{tabular}{|c|} 
Year of \\
publication
\end{tabular}} & \multirow[b]{2}{*}{$\begin{array}{c}\text { Type } \\
\text { of } \\
\text { study }\end{array}$} & \multirow[b]{2}{*}{ Groups } & \multicolumn{3}{|c|}{ Treatment provided } & \multirow[b]{2}{*}{$\left|\begin{array}{c|}\mathrm{N} \\
\text { patients }\end{array}\right|$} & \multirow[b]{2}{*}{$\underset{\text { implants }}{\mathbf{N}}$} & \multirow[b]{2}{*}{$\left|\begin{array}{c}\text { Follow- } \\
\text { up } \\
\text { (months) }\end{array}\right|$} & \multirow[b]{2}{*}{\begin{tabular}{|c|} 
Diagnosis \\
$\begin{array}{c}\text { Mucositis/ } \\
\text { peri- } \\
\text { implantitis }\end{array}$
\end{tabular}} & \multicolumn{7}{|c|}{ Treatment outcomes } & \multirow[b]{2}{*}{$\begin{array}{l}\text { Microbiological } \\
\text { results }\end{array}$} & \multirow[b]{2}{*}{ Conclusions } \\
\hline & & & & $\begin{array}{c}\text { Self- } \\
\text { performed }\end{array}$ & \multicolumn{2}{|c|}{$\begin{array}{l}\text { Professionally- } \\
\text { delivered }\end{array}$} & & & & & $\begin{array}{c}\text { PDs } \\
\text { reduction } \\
\text { Mean (SD), } \\
\mathrm{mm}\end{array}$ & $\begin{array}{c}\text { Radiographic } \\
\text { MBL } \\
\text { changes } \\
\text { Mean (SD), } \\
\text { mm }\end{array}$ & $\begin{array}{c}\text { BOP } \\
\text { changes } \\
\text { Mean (SD), } \\
\text { mm }\end{array}$ & $\begin{array}{l}\text { Exudate } \\
\text { changes }\end{array}$ & $\mid \begin{array}{c}\text { PI or MPI } \\
\text { (SD) changes }\end{array}$ & $\underset{\substack{\text { Mean }(\text { SD) } \\
\mathbf{m m}}}{\mathbf{C A L}}$ & $\begin{array}{c}\text { Recession } \\
\text { Mean (SD), } \\
\text { mm }\end{array}$ & & \\
\hline \multirow[b]{2}{*}{$\begin{array}{l}\text { Arisan et } \\
\text { al. [20] }\end{array}$} & \multirow[b]{2}{*}{2015} & \multirow[b]{2}{*}{ RCT } & Control & $\mathrm{OHI}$ & \multicolumn{2}{|l|}{ MD } & 5 & 24 & \multirow[b]{2}{*}{6} & \multirow[b]{2}{*}{ Peri-implantitis } & $\begin{array}{l}4.38(0.42) \\
\text { to } \\
4.17(0.41)\end{array}$ & $\begin{array}{l}2.35(0.56) \\
\text { to } \\
2.63(0.53)\end{array}$ & 100 to $100 \%$ & $\mathrm{NA}$ & 91.7 to $41.7 \%$ & $\mathrm{NA}$ & $\mathrm{NA}$ & \multirow[b]{2}{*}{$\begin{array}{l}\text { No changes in microbiota } \\
\text { after } 1 \text { month }\end{array}$} & \multirow[b]{2}{*}{$\begin{array}{c}\text { Laser does not provide any } \\
\text { additional benefit when compared } \\
\text { to SRP alone. }\end{array}$} \\
\hline & & & Test & OHI & \multicolumn{2}{|c|}{$\begin{array}{c}\mathrm{MD}+\text { diode laser } 810 \mathrm{~nm} \\
\text { (energy density: } 3 \mathrm{~J} / \mathrm{cm}^{2} ; \\
\text { time: } 1 \mathrm{~min} ; \\
\text { power density: } 400 \mathrm{~mW} / \mathrm{cm}^{2} ; \\
\text { energy: } 1.5 \mathrm{~J} ; \\
\text { spot diameter: } 1 \mathrm{~mm} \text { ) }\end{array}$} & 5 & 24 & & & $\begin{array}{l}4.71(0.67) \\
\quad \text { to } \\
4.54(0.74)\end{array}$ & $\begin{array}{l}2.13(0.47) \\
\text { to } \\
2.79(0.48)\end{array}$ & 100 to $95.8 \%$ & $\mathrm{NA}$ & 91.7 to $54.2 \%$ & NA & $\mathrm{NA}$ & & \\
\hline \multirow{2}{*}{$\begin{array}{l}\text { Bassetti } \\
\text { et al. [21] }\end{array}$} & \multirow{2}{*}{2014} & \multirow{2}{*}{ RCT } & Control & Instructions & \multicolumn{2}{|c|}{$\begin{array}{l}\mathrm{MD}+\text { Airpolishing } \\
+3 \% \text { hydrogen peroxide } \\
\text { irrigation } \\
+ \text { Arestin }\end{array}$} & 20 & 20 & \multirow{2}{*}{12} & \multirow{2}{*}{ Peri-implantitis } & $\begin{array}{l}4.39(0.77) \\
\text { to } \\
3.83(0.85)\end{array}$ & $\mathrm{NA}$ & $\begin{array}{c}\text { Sites: } \\
4.41(1.47) \\
\text { to } \\
1.55(1.26) \\
(65 \% \\
\text { reduction })\end{array}$ & $\mathrm{NA}$ & $\begin{array}{l}0.21(0.27) \\
\text { to } \\
0(0)\end{array}$ & $\begin{array}{c}2.72(0.72) \\
\text { to } \\
2.41(0.7)\end{array}$ & $\begin{array}{l}1.68(1.04) \\
\text { to } \\
1.41(1.18)\end{array}$ & $\begin{array}{c}\text { P. gingivalis, T. forsythia, T. } \\
\text { denticola, C. rectus, F nucleatum } \\
\text { and E. corrodens demonstrated } \\
\text { statistically significant decrease } \\
\text { from baseline. }\end{array}$ & \multirow{2}{*}{$\begin{array}{l}\text { Similar results clinically and } \\
\text { microbiologically for both. } \\
\text { Complete disease resolution not } \\
\text { routinely achieved. }\end{array}$} \\
\hline & & & Test & & \multicolumn{2}{|c|}{$\begin{array}{c}\mathrm{MD}+\text { Airpolishing }+\mathrm{PDT} \\
(660 \mathrm{~nm}, \text { power density } 100 \mathrm{~mW}) \\
+3 \% \text { hydrogen peroxide } \\
\text { irrigation }\end{array}$} & 20 & 20 & & & $\begin{array}{l}4.19(0.55) \\
\text { to } \\
4.08(0.81)\end{array}$ & $\mathrm{NA}$ & $\begin{array}{c}\text { Sites: } \\
4.03(1.66) \\
\text { to } \\
1.74(1.37) \\
(57 \% \\
\text { reduction })\end{array}$ & NA & $\begin{array}{l}0.13(0.21) \\
\text { to } \\
0.01(0.04)\end{array}$ & $\begin{array}{l}2.66(0.73) \\
\text { to } \\
2.58(0.94)\end{array}$ & $\begin{array}{c}1.53(0.91) \\
\text { to } \\
1.5(0.86)\end{array}$ & $\begin{array}{l}\text { No statistically significant } \\
\text { difference with the exception for } \\
\text { F. nucleatum. }\end{array}$ & \\
\hline \multirow{2}{*}{$\begin{array}{l}\text { Esposito } \\
\text { et al. [22] }\end{array}$} & \multirow{2}{*}{2013} & \multirow{2}{*}{ RCT } & Control & $\begin{array}{l}\text { In surgery group: } \\
\text { CHX mouthwash } \\
0.12 \% \text { for } 1 \mathrm{~min} \\
\text { twice a day for } 2\end{array}$ & \multirow{2}{*}{\begin{tabular}{|c} 
Bone loss between \\
$3-5 \mathrm{~mm}:$ \\
implant surface \\
DE bone loss $>5$ \\
mm: surgery, full- \\
thickness flap and \\
DE, \\
remove all \\
granulation tissue
\end{tabular}} & $\begin{array}{c}\text { No adjunct } \\
\text { treatment }\end{array}$ & 40 & 100 & 12 & Dori inolitit & $\begin{array}{l}6.45(2.15) \\
\text { to } \\
5.5(1.94)\end{array}$ & $\begin{array}{l}4.9(2.07) \\
\text { to } \\
5.03(2.51)\end{array}$ & $\begin{array}{l}\text { Bleeding } \\
\text { scores: } \\
2.68(1.25) \\
\text { to } \\
1.28(1.11)\end{array}$ & $\mathrm{NA}$ & $\begin{array}{c}\text { Mean plaque } \\
\text { scores: } \\
2.15(1.64) \\
\text { to } \\
0.93(0.94)\end{array}$ & $\mathrm{NA}$ & $\mathrm{NA}$ & $\mathrm{NA}$ & $\begin{array}{l}\text { Adjunctive use of LAD therapy } \\
\text { with mechanical debridement } \\
\text { did not timprove ann clinical }\end{array}$ \\
\hline & & & Test & $\begin{array}{c}\text { All gourps: gentle } \\
\text { wiping with a soft } \\
\text { brush }\end{array}$ & & $\begin{array}{c}\text { LAD } \\
\text { (FotoSan } \\
630 \\
\text { instrument) }\end{array}$ & 40 & 101 & & & $\begin{array}{l}6.23(1.62) \\
\text { to } \\
5.14(1.83)\end{array}$ & $\begin{array}{l}4.5(1.75) \\
\text { to } \\
4.5(1.67)\end{array}$ & $\begin{array}{c}\begin{array}{c}\text { Bleeding } \\
\text { scores: } \\
2.95(1.32) \text { to } \\
1.35(1.32)\end{array}\end{array}$ & $\mathrm{NA}$ & $\begin{array}{c}\text { Mean plaque } \\
\text { scores: } \\
2.18(1.53) \\
\text { to } \\
0.89(0.94)\end{array}$ & $\mathrm{NA}$ & $\mathrm{NA}$ & $\mathrm{NA}$ & $\begin{array}{l}\text { mechanical cleaning alone up to } 1 \\
\text { year after treatment. }\end{array}$ \\
\hline Hallstrom & 2012 & RCT & Control & $\mathrm{OHI}$ & $\begin{array}{r}\text { MD with titaniu } \\
+ \text { polishi }\end{array}$ & g curettes & 21 & $\mathrm{NA}$ & 6 & Mucositis & $\begin{array}{c}4.6(0.9) \\
\text { to } \\
4.1(1.2)\end{array}$ & $\mathrm{NA}$ & $\begin{array}{l}\text { Full mouth } \\
\text { BOP \%: } \\
24.2(16.7) \\
\text { to } \\
18.4(17.4)\end{array}$ & $\mathrm{NA}$ & $\begin{array}{l}\text { Mean PI at } \\
\text { implant \%: } \\
22(29.2) \\
\text { to } \\
17.9(28.4)\end{array}$ & $\mathrm{NA}$ & $\mathrm{NA}$ & $\begin{array}{l}\text { No significant differences in } \\
\text { bacterial count. Statistical } \\
\text { analysis by intent to treat failed to }\end{array}$ & $\begin{array}{l}\text { No short-term differences } \\
\text { between - groups. The clinical } \\
\text { improvements observed at } 6 \\
\text { months may be attributed to }\end{array}$ \\
\hline & & & Test & & $\begin{array}{r}\text { MD with titaniu } \\
\text { + polishing } \\
\text { (Azithromycin }{ }^{2} \\
\text { and } \\
250 \mathrm{mg} \text { days }\end{array}$ & $\begin{array}{l}\text { curettes } \\
\text { ABX } \\
0 \mathrm{mg} \text { day } 1 \\
\text { - 4). }\end{array}$ & 22 & $\mathrm{NA}$ & & & $\begin{array}{c}4.4(1) \\
\text { to } \\
3.5(1.1)\end{array}$ & $\mathrm{NA}$ & $\begin{array}{l}\text { Full mouth } \\
\text { BOP \%: } \\
28.2(20.6) \\
\text { to } \\
10.1(6.9)\end{array}$ & $\mathrm{NA}$ & $\begin{array}{l}\text { Mean PI at } \\
\text { implatan \%: } \\
33.7(35.5) \\
\text { to } \\
6.8(13.8)\end{array}$ & $\mathrm{NA}$ & $\mathrm{NA}$ & $\begin{array}{l}\text { comparing baseline data with all } \\
\text { other time points. }\end{array}$ & $\begin{array}{l}\text { No evidence for the use of } \\
\text { systemic antibiotics in treatment } \\
\text { of peri-implant mucositis. }\end{array}$ \\
\hline
\end{tabular}
available; $\mathrm{OHI}=$ oral hygiene instructions; $\mathrm{PD}=$ probing depth; $\mathrm{PDT}$ = photodynamic therapy; $\mathrm{PI}=$ plaque index; $\mathrm{PR}=$ prospective study; $\mathrm{RB}=$ radiographic bone; $\mathrm{RCT}=$ randomized controlled trials; $\mathrm{SD}=$ standard deviation 


\section{Table 1B. Characteristics of the included articles}

\begin{tabular}{|c|c|c|c|c|c|c|c|c|c|c|c|c|c|c|c|c|c|c|}
\hline \multirow[b]{2}{*}{ Study } & \multirow[b]{2}{*}{\begin{tabular}{|c|} 
Year of \\
publication
\end{tabular}} & \multirow[b]{2}{*}{\begin{tabular}{|l|} 
Type of \\
nstudy
\end{tabular}} & \multirow[b]{2}{*}{ Groups } & \multicolumn{2}{|c|}{ Treatment provided } & \multirow[b]{2}{*}{$\begin{array}{c}\mathbf{N} \\
\text { patients }\end{array}$} & \multirow[b]{2}{*}{ implants } & \multirow[b]{2}{*}{ s $\left.\begin{array}{c}\text { Follow- } \\
\text { up } \\
\text { (months) }\end{array}\right)$} & \multirow{2}{*}{\begin{tabular}{|c|} 
Diagnosis \\
$\begin{array}{c}\text { Mucositis/ } \\
\text { peri- } \\
\text { implantitis }\end{array}$
\end{tabular}} & \multicolumn{7}{|c|}{ Treatment outcomes } & \multirow[b]{2}{*}{$\begin{array}{l}\text { Microbiological } \\
\text { results }\end{array}$} & \multirow[b]{2}{*}{ Conclusions } \\
\hline & & & & $\begin{array}{c}\begin{array}{c}\text { Self- } \\
\text { performed }\end{array} \\
\text { por }\end{array}$ & $\begin{array}{l}\text { Professionally- } \\
\text { delivered }\end{array}$ & & & & & $\begin{array}{c}\text { PDs } \\
\text { reduction } \\
\text { Mean (SD), } \\
\text { mm }\end{array}$ & $\begin{array}{c}\text { Radiographic } \\
\text { MBL changes } \\
\text { Mean (SD), mm }\end{array}$ & $\begin{array}{l}\text { BOP changes } \\
\text { Mean (SD), mm }\end{array}$ & $\begin{array}{l}\text { Exudate } \\
\text { changes }\end{array}$ & $\left|\begin{array}{c}\text { PI or } \\
\text { MPI (SD) } \\
\text { changes }\end{array}\right|$ & $\underset{\substack{\text { CAan (SD) } \\
\mathrm{mm}}}{\text { CAL }}$ & $\left\{\begin{array}{c}\text { Recession } \\
\text { Mean (SD) } \\
\mathrm{mm}\end{array}\right.$ & & \\
\hline \multirow{2}{*}{$\begin{array}{c}\text { John et al. } \\
\text { [24] }\end{array}$} & \multirow[b]{2}{*}{2015} & \multirow[b]{2}{*}{ RCT } & AAD & $\begin{array}{l}\text { OHI on } 2 \text { to } 4 \\
\text { appointments }\end{array}$ & \begin{tabular}{|c|}
$\begin{array}{c}\text { Submucosal AAD } \\
\text { employed with amino } \\
\text { acid glycine powder (Air- } \\
\text { Flowß Perio Powder, } \\
\text { EMS) }\end{array}$ \\
\end{tabular} & 12 & 18 & \multirow[b]{2}{*}{12} & \multirow{2}{*}{$\begin{array}{c}\text { Peri- } \\
\text { implantitis }\end{array}$} & $\begin{array}{c}3.7(1) \\
\text { to } \\
3.2(1.1)\end{array}$ & $\mathrm{NA}$ & $\begin{array}{c}99(4.1) \\
\text { to } \\
57.8(30.7) \%\end{array}$ & NA & $\begin{array}{c}1.2(1.1) \\
\text { to } \\
1.8(1.1)\end{array} \mid$ & $\begin{aligned} & 5.2(1.9) \text { to } \\
& 4.6(1.8)\end{aligned}$ & $\begin{array}{l}1.5(1.4) \\
\text { to } \\
1.4(1.3)\end{array}$ & NA & \multirow{2}{*}{$\begin{array}{c}\text { Both treatments resulted in } \\
\text { comparable but limited CAL gains } \\
\text { at } 12 \text { months. AAD was associated } \\
\text { with significantly higher BOP } \\
\text { decrease than MDA. }\end{array}$} \\
\hline & & & $\begin{array}{l}\text { MD (carbon } \\
\text { curettes } \\
\text { + local } \\
\text { antiseptic } \\
\text { therapy) }\end{array}$ & $\begin{array}{l}\text { OHI on } 2 \text { to } 4 \\
\text { appointments }\end{array}$ & $\begin{array}{c}\text { MD was performed using } \\
\text { carbon curets followed by } \\
\text { pocket irrigation with a } \\
0.1 \% \text { CHX solution and } \\
\text { submucosal application of } \\
1 \% \text { CHX gel }\end{array}$ & 13 & 18 & & & $\begin{array}{l}3.9(1.1) \\
\text { to } \\
3.5(1.2)\end{array}$ & NA & $\begin{array}{l}94.7(13.7) \\
\text { to } \\
78.1(30) \%\end{array}$ & NA & $\begin{array}{c}1.2(1) \\
\text { to } \\
0.9(0.7)\end{array}$ & $\begin{array}{l}5(1.5) \\
\text { to } \\
4.5(1.3)\end{array}$ & $\begin{array}{c}1(1.1) \\
\text { to } \\
0.9(1.1)\end{array}$ & $\mathrm{NA}$ & \\
\hline \multirow{2}{*}{$\begin{array}{c}\text { Machtei et } \\
\text { al. [25] }\end{array}$} & \multirow{2}{*}{2012} & \multirow{2}{*}{$\begin{array}{l}\text { Double- } \\
\text { blind } \\
\text { RCT }\end{array}$} & Control & \begin{tabular}{|c|} 
OHI \\
patients were given \\
sodium fluoride \\
toothpaste
\end{tabular} & \begin{tabular}{|c|} 
Surface $\mathrm{MD}+$ \\
biodegradable crosslinked \\
gelatin matrix chip \\
(placebo)
\end{tabular} & 30 & 37 & \multirow{2}{*}{6} & \multirow{2}{*}{$\begin{array}{c}\text { Peri- } \\
\text { implantitis }\end{array}$} & $\begin{array}{c}7.21 \\
\text { to } \\
5.48\end{array}$ & NA & 100 to $42.5 \%$ & NA & NA & $\begin{array}{l}7.63(0.3) \\
\text { to } \\
5.94(0.3)\end{array}$ & NA & NA & \multirow{2}{*}{$\begin{array}{l}\text { Substantial reduction in PD, gain in } \\
\text { CAL and reduction in BOP in sites } \\
\text { with peri-implantitis. }\end{array}$} \\
\hline & & & Test & \begin{tabular}{|c|} 
OHI \\
patients were given \\
sodium fluoride \\
toothpaste
\end{tabular} & $\begin{array}{c}\text { Surface MD+ matrix } \\
\text { containing } 2.5 \mathrm{mg} \mathrm{CHX} \\
\text { chips }\end{array}$ & 30 & 40 & & & $\begin{array}{c}7.60 \\
\text { to } \\
5.47\end{array}$ & NA & 100 to $59 \%$ & NA & NA & \begin{tabular}{|l}
$7.88(0.2)$ \\
to \\
$5.7(0.3)$
\end{tabular} & NA & NA & \\
\hline \multirow{2}{*}{$\begin{array}{l}\text { Persson et } \\
\text { al. [26] }\end{array}$} & \multirow{2}{*}{2011} & \multirow{2}{*}{ RCT } & Er:YAG laser & $\begin{array}{l}\text { OHI and patients } \\
\text { received a sonic } \\
\text { toothbrush }\end{array}$ & $\begin{array}{c}\text { (Er:YAG) laser: } 100 \mathrm{~mJ} / \\
\text { pulse and } 10 \mathrm{~Hz}(12.7 \mathrm{~J} / \\
\left.\mathrm{cm}^{2}\right)\end{array}$ & 21 & 55 & \multirow{2}{*}{6} & \multirow{2}{*}{$\begin{array}{c}\text { Peri- } \\
\text { implantitis }\end{array}$} & \begin{tabular}{|c|} 
PD \\
reductions: \\
$0.9(0.8)$ \\
\end{tabular} & \begin{tabular}{|c|} 
Statistical analyses failed \\
to demonstrate differences \\
in bone-level changes \\
between baseline and 6 \\
months
\end{tabular} & $\begin{array}{c}\text { Statistical analyses also } \\
\text { failed to demonstrate } \\
\text { differences in the BOP at } 6 \\
\text { weeks after treatment }\end{array}$ & NA & NA & NA & NA & \multirow{2}{*}{\begin{tabular}{|c|} 
Both treatments failed to \\
reduce bacterial counts at \\
6 months. Porphyromonas \\
gingivalis counts \\
were higher in cases \\
with progressive peri- \\
implantitis \\
ing
\end{tabular}} & \multirow{2}{*}{$\begin{array}{l}\text { At } 1 \text { month, } P \text {. aeruginosa, } S \text {. } \\
\text { aurreus, and S. anaerobous were } \\
\text { reduced in the air-abrasive group, } \\
\text { and Fussobacterium mspp. were } \\
\text { reduced in the laser group. } 6 \\
\text { month data demonstrated that both } \\
\text { methods failed to reduce bacterial } \\
\text { counts. Clinical improvements were } \\
\text { limited. }\end{array}$} \\
\hline & & & $\begin{array}{l}\text { AAD } \\
\text { subgingival } \\
\text { polishing }\end{array}$ & \begin{tabular}{|} 
OHI patients received \\
a sonic toothbrush
\end{tabular} & $\begin{array}{l}\text { AAD subgingival } \\
\text { polishing for } 15 \text { sec in } \\
\text { each position }\end{array}$ & 21 & 45 & & & $\begin{array}{c}\text { PD } \\
\text { reductions: } \\
0.8(0.5) \\
\end{array}$ & \begin{tabular}{|c|} 
Statistical analyses failed \\
to demonstrate differences \\
in bone-level changes \\
between baseline and 6 \\
months \\
\end{tabular} & $\begin{array}{c}\text { Statistical analyses also } \\
\text { failed to demonstrate } \\
\text { differences in the BOP at } 6 \\
\text { weeks after treatment }\end{array}$ & NA & NA & NA & NA & & \\
\hline \multirow{2}{*}{$\begin{array}{c}\text { Riben- } \\
\text { Grundstrom } \\
\text { et al. [27] }\end{array}$} & \multirow{2}{*}{2015} & \multirow{2}{*}{ RCT } & $\begin{array}{l}\text { Glycine } \\
\text { powder air- } \\
\text { polishing } \\
\text { group }\end{array}$ & OHI & \begin{tabular}{|c|} 
Glycine powder air- \\
polishing was performed \\
at baseline, 3 and 6 \\
months. Supragingival \\
DE was provided at \\
month 9 and 12 .
\end{tabular} & 18 & NA & 12 & Muriti & $\mathrm{NA}$ & NA & $43.9(7.3)$ to $12.1(3.8)$ & $\begin{array}{l}\text { No } \\
\text { differences } \\
\text { were } \\
\text { found }\end{array}$ & s $\begin{array}{c}\text { Implant } \\
25.5(6.8) \\
\text { to } \\
5.6(3.8)\end{array} \mid$ & NA & $\begin{array}{c}\text { No } \\
\text { differences } \\
\text { were } \\
\text { found }\end{array}$ & NA & $\begin{array}{l}\text { Non-surgical treatment with a } \\
\text { glycine powder air-polishing or } \\
\text { ultrasonic device is effective in }\end{array}$ \\
\hline & & & $\begin{array}{l}\text { Ultrasonic } \\
\text { group }\end{array}$ & OHI & $\begin{array}{c}\text { Cleaning with ultrasonic } \\
\text { was performed at } \\
\text { baseline, } 3 \text { and } 7 \text { months. } \\
\text { Supragingival MD was } \\
\text { provided at month } 9 \text { and } \\
12 . \\
\end{array}$ & 18 & NA & 12 & Nucostis & NA & NA & $53.7(7.9)$ to $18.6(6.4)$ & $\begin{array}{l}\text { No } \\
\text { differences } \\
\text { were } \\
\text { found }\end{array}$ & s $\begin{array}{c}\text { Implant } \\
24.1(6.6) \\
\text { to } \\
7.4(6.4)\end{array}$ & NA & $\begin{array}{c}\text { No } \\
\text { differences } \\
\text { were } \\
\text { found }\end{array}$ & NA & $\begin{array}{c}\text { reducing inflammation and number } \\
\text { of peri-implant pockets subject to } \\
\text { patient compliance }\end{array}$ \\
\hline $\begin{array}{c}\text { Swierkot et } \\
\text { al. [28] }\end{array}$ & 2013 & RCT & $\begin{array}{l}\text { Sonic } \\
\text { toothbrush } \\
\text { group }\end{array}$ & $\begin{array}{c}\text { Brush } 2 \text { min twice } \\
\text { daily with } \\
\text { toothpaste, brush } \\
\text { their teeth with sonic } \\
\text { tooth brush according } \\
\text { to the manufacturer's } \\
\text { instructions }\end{array}$ & NA & 35 & $\begin{array}{l}\text { Mean } \\
\text { count: } \\
4.19\end{array}$ & 12 & $\mid \begin{array}{c}\text { No peri- } \\
\text { implantitis, } \\
22 \% \text { mucositis }\end{array}$ & $\begin{array}{c}3.4(0.88) \\
\text { to } \\
3.4(0.8)\end{array}$ & NA & $0.22(0.3)$ to $0.27(0.26)$ & NA & $\left.\begin{array}{c}0.86(0.73) \\
\text { to } \\
1(0.79)\end{array}\right)$ & $\left|\begin{array}{r}4.64(1.63) \\
\text { to } \\
5.1(1.78)\end{array}\right|$ & $\mid \begin{array}{c}1.23(1.33) \\
\text { to } \\
1.7(1.48)\end{array}$ & \begin{tabular}{|} 
After 12 months, both \\
groups exhibited a small \\
increase in total bacterial \\
load at implants and teeth. \\
$P$ gingivalis, $P$ micra and \\
D. pneumosintes were
\end{tabular} & $\begin{array}{l}\text { No difference between sonic and } \\
\text { manual tooth brushing for plaque } \\
\text { reduction at implants and teeth. } \\
\text { Sonic and manual toothbrushes } \\
\text { maintained clinical, microbiological } \\
\text { and immunological parameters over }\end{array}$ \\
\hline & & & $\begin{array}{l}\text { Manual } \\
\text { toothbrush } \\
\text { group }\end{array}$ & $\begin{array}{c}\text { Brush } 2 \text { min twice } \\
\text { daily with toothpaste, } \\
\text { modified Bass } \\
\text { technique }\end{array}$ & NA & 36 & $\begin{array}{l}\text { Mean } \\
\text { count: } \\
4.32\end{array}$ & & $\left|\begin{array}{c}\text { No peri- } \\
\text { implantitis, } \\
19 \% \text { mucositis }\end{array}\right|$ & $\begin{array}{r}3.13(0.75) \\
\text { to } \\
3.13(0.78)\end{array}$ & NA & \begin{tabular}{|l|l}
$0.19(0.28)$ to $0.28(0.38)$ \\
\end{tabular} & NA & $\begin{array}{r}0.56(0.52) \\
\text { to } \\
0.92(0.93)\end{array} \mid$ & $\left|\begin{array}{l}4.41(1.65) \\
\text { to } \\
4.43(1.28)\end{array}\right|$ & $\begin{array}{c}1.28(1.26) \\
\text { to } \\
1.29(0.85)\end{array}$ & $\begin{array}{c}\text { nearly every examination } \\
\text { time for implant and teeth } \\
\text { groups. }\end{array}$ & $\begin{array}{l}\text { a period of } 12 \text { months at implants } \\
\text { and teeth with no signs of soft titsue } \\
\text { damage or technical complications. }\end{array}$ \\
\hline
\end{tabular}

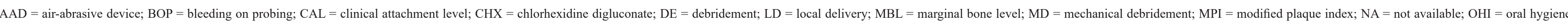
instructions; $\mathrm{PD}=$ probing depth; $\mathrm{PI}=$ plaque index; $\mathrm{RB}=$ radiographic bone; $\mathrm{RCT}$ = randomized controlled trials; $\mathrm{SD}=$ standard deviation. 
Table 1C. Characteristics of the included articles

\begin{tabular}{|c|c|c|c|c|c|c|c|c|c|c|c|c|c|c|c|c|c|c|}
\hline \multirow[b]{2}{*}{ Study } & \multirow[b]{2}{*}{$\begin{array}{c}\text { Year of } \\
\text { publication }\end{array}$} & \multirow[b]{2}{*}{$\begin{array}{c}\text { Type of } \\
\text { study }\end{array}$} & \multirow[b]{2}{*}{ Groups } & \multicolumn{2}{|c|}{ Treatment provided } & \multirow[b]{2}{*}{$\left|\begin{array}{c}\mathbf{N} \\
\text { patients }\end{array}\right|$} & \multirow[b]{2}{*}{ implants } & \multirow[b]{2}{*}{$s \mid \begin{array}{l}\text { Follow-up } \\
\text { (months) }\end{array}$} & \multirow[b]{2}{*}{\begin{tabular}{|c|} 
Diagnosis \\
$\begin{array}{c}\text { Mucositis/ } \\
\text { peri- } \\
\text { implantitis }\end{array}$
\end{tabular}} & \multicolumn{7}{|c|}{ Treatment outcomes } & \multirow[b]{2}{*}{$\left.\right|_{\text {results }} ^{\text {Microbiological }}$} & \multirow[b]{2}{*}{ Conclusions } \\
\hline & & & & $\begin{array}{c}\text { Self- } \\
\text { performed }\end{array}$ & $\begin{array}{l}\text { Professionally- } \\
\text { delivered }\end{array}$ & & & & & \begin{tabular}{|c|} 
PDs \\
reduction \\
Mean (SD), \\
mm
\end{tabular} & \begin{tabular}{|c|} 
Radiographic \\
MBL \\
changes \\
Mean (SD), \\
mm \\
\end{tabular} & $\begin{array}{l}\text { BOP changes } \\
\text { Mean (SD), } \\
\text { mm }\end{array}$ & \begin{tabular}{|l} 
Exudate \\
changes
\end{tabular} & $\begin{array}{c}\text { PI or MPI (SD) } \\
\text { changes }\end{array}$ & $\underset{\substack{\text { Mean (SD) } \\
\text { mm }}}{\text { CAL }}$ & $\begin{array}{c}\text { Recession } \\
\text { Mean (SD), } \\
\mathrm{mm}\end{array}$ & & \\
\hline $\begin{array}{l}\text { Gomes et } \\
\text { al. [29] }\end{array}$ & 2015 & $\begin{array}{c}\text { Single-arm } \\
\text { cohort }\end{array}$ & Test & $\begin{array}{c}\text { Multi-tufted TB, dental } \\
\text { floss and/or interdental } \\
\text { TB. } \\
\text { Non-therapeutic } \\
\text { fluoride toothpaste }\end{array}$ & $\begin{array}{l}\text { Weekly supragingival/ } \\
\text { supramucosal } \\
\text { MD for } 1 \text { month, } \\
\text { then every } 3 \text { months }\end{array}$ & 14 & 59 & 13 & Mucositis & $\begin{array}{r}3.62(0.31) \\
\text { to } \\
2.55(0.16)\end{array}$ & NA & $\begin{array}{c}\text { Sites: } \\
54.05(5.88) \\
\text { to } \\
4.96(1.78) \%\end{array}$ & NA & $\begin{array}{l}18.98(5.89) \\
\text { to } \\
2.7(1.47) \% \\
\text { /390 days }\end{array}$ & NA & NA & NA & $\begin{array}{l}\text { The supragingival-supramucosal } \\
\text { biofilm control benefited both teeth } \\
\text { and implants }\end{array}$ \\
\hline $\begin{array}{l}\text { Corbella } \\
\text { et al. [30] }\end{array}$ & 2011 & $\begin{array}{c}\text { Non- } \\
\text { controlled } \\
\text { prospective } \\
\text { study }\end{array}$ & \begin{tabular}{|c|} 
Mucositis \\
Peri- \\
implantitis
\end{tabular} & $\mid \begin{array}{c}\begin{array}{c}\text { CHX } 0.2 \% \text { mouthwash } \\
\text { twice a day for 10 } \\
\text { days, interdental } \\
\text { brushes }\end{array} \\
\begin{array}{c}\text { CHX } 0.2 \% \\
\text { mouthwash, interdental } \\
\text { brushes }\end{array}\end{array}$ & \begin{tabular}{|c|}
$\begin{array}{c}\text { Powered and manual } \\
\text { devices MD }\end{array}$ \\
$\begin{array}{c}\text { Powered and manual } \\
\text { devices } \\
+ \text { LD of CHX 1\% } \\
+ \text { Sx MD }\end{array}$ \\
\end{tabular} & 61 & 244 & 60 & Peri-implantiti & $\begin{array}{l}2.2(0.87) \\
\text { to } \\
2.46(0.5)\end{array}$ & NA & \begin{tabular}{l}
\multicolumn{1}{c}{ Sites: } \\
Index 0: 88.2 to $100 \%$ \\
Index 1: 1.4 to $0 \%$ \\
Index 2: 10.4 to $0 \%$ \\
Index 3: 0 to $0 \%$
\end{tabular} & NA & $\begin{array}{l}\text { Sites/6 and } 58 \text { months } \\
\text { Index 0: } 58.3 \% \text { to } 88.5 \% \text {; } \\
\text { IIdex 1:2.8 to } 7.3 \% ; \\
\text { Index } 2: 9.7 \text { to } 1 \% \% \\
\text { Index } 3: 29.2 \text { to } 3.2 \%\end{array}$ & NA & NA & NA & $\begin{array}{l}\text { Systematic hygienic protocol is } \\
\text { effective in keeping low the incidenc } \\
\text { of peri-implant mucositis as well as i } \\
\text { controlling plaque accumulation and } \\
\text { clinical attachment loss. }\end{array}$ \\
\hline $\begin{array}{l}\text { Costa et } \\
\text { al. [31] }\end{array}$ & 2012 & $\begin{array}{c}\text { Prospective } \\
\text { study }\end{array}$ & Control & $\mathrm{OHI}$ & $\begin{array}{c}\text { At least } 5 \mathrm{SC}, \\
\text { coronal prophylaxis }\end{array}$ & 39 & 183 & 60 & Mucositis & $\begin{array}{c}5.9 \% \text { sites } \\
\text { with } \\
\mathrm{PD} \geq 5 \mathrm{~mm}\end{array}$ & $\begin{array}{c}17.9 \% \\
\text { implants show } \\
\text { BL }\end{array}$ & $\begin{array}{c}\text { Sites: } \\
50.2 \text { to } 62.6 \%\end{array}$ & NA & $\begin{array}{c}1.4(0.6) \\
\text { to } \\
1.4(0.7)\end{array}$ & $\mid \begin{array}{c}\% \text { sites }>3 \mathrm{~mm} \\
\mathrm{CAL} \\
14.9(16.7) \\
\text { to } \\
22.7(23.2) \\
\\
\% \text { sites }>3 \mathrm{~mm} \\
\mathrm{CAL} \\
15.9(19) \\
\text { to } \\
20.1(23)\end{array}$ & NA & NA & $\begin{array}{l}\text { The absence of preventive } \\
\text { maintenance in individuals with pre- } \\
\text { existing peri-implant mucositis was } \\
\text { associated with a high incidence of } \\
\text { peri-implantitis. }\end{array}$ \\
\hline $\begin{array}{c}\text { Deppe et } \\
\text { al. [32] }\end{array}$ & 2013 & $\begin{array}{c}\begin{array}{c}\text { Prospective } \\
\text { study }\end{array} \\
\text {. }\end{array}$ & \begin{tabular}{|l|} 
Moderate \\
bone loss \\
\end{tabular} & $\begin{array}{l}\text { OHI, plaque control } \\
\text { with use of CHX } \\
\text { solution }(0.3 \%)\end{array}$ & $\begin{array}{c}\text { Calculus removal } \\
+ \text { antimicrobial PDT }\end{array}$ & 16 & 10 & 6 & Peri-implantitis & $\begin{array}{l}3.3(0.8) \\
\text { to } \\
2.9(0.5)\end{array}$ & $\begin{array}{c}3.9(0.8) \\
\text { to } \\
3.6(0.8) \mathrm{mm}\end{array}$ & \begin{tabular}{|c|} 
Sulcus bleeding index: \\
$1.8(1.3)$ \\
to \\
$1.1(0.9)$ \\
Sulcus bleeding index: \\
$1.5(1.2)$ \\
to \\
$1.3(1.1)$ \\
\end{tabular} & NA & NA & $\begin{array}{l}6.7(0.9) \\
\text { to } \\
8.1(0.9)\end{array}$ & $\begin{array}{l}0.9(1.2) \\
\text { to } \\
1.6(1.2)\end{array}$ & NA & $\begin{array}{c}\text { Non-surgical PDT could stop bone } \\
\text { resorption in moderate peri-implant } \\
\text { defects but not in severe defects. } \\
\text { marginal tissue recession was not } \\
\text { significantly different in both groups. }\end{array}$ \\
\hline $\begin{array}{l}\text { Schwarz } \\
\text { et al. [33] }\end{array}$ & 2015 & $\begin{array}{c}\text { Prospective } \\
\text { case series }\end{array}$ & \begin{tabular}{|c|} 
MD + \\
local \\
antiseptic \\
(MD + \\
CHX)
\end{tabular} & $\mathrm{OHI}$ & \begin{tabular}{|c|} 
Supragingival calculus \\
removal \\
and supramucosal/ \\
gingival professional \\
implant/ \\
tooth cleaning \\
+ laser tx
\end{tabular} & 17 & 24 & 6 & Peri-implantitis & $\begin{array}{l}3.4(0.5) \\
\text { to } \\
3.3(0.5)\end{array}$ & NA & $\begin{array}{c}45(18.5) \\
\text { to } \\
14.2(11.6) \%\end{array}$ & NA & $\begin{array}{l}0.7(0.6) \\
\text { to } \\
0.4(0.5)\end{array}$ & NA & NA & NA & $\begin{array}{l}\text { Non-surgical treatment of either peri- }- \text { - } \\
\text { implant mucositis using MD }+ \text { CHX } \\
\text { or peri-implantitis using laser therapy } \\
\text { at zirconia implants was associated } \\
\text { with significant short-term clinical } \\
\text { improvements. A complete disease } \\
\text { resolution, however, was not achieved } \\
\text { in the majority of the patients. }\end{array}$ \\
\hline
\end{tabular}

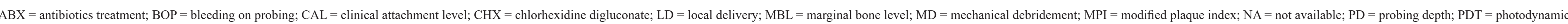
therapy; $\mathrm{PI}=$ plaque index; $\mathrm{RB}=$ radiographic bone; $\mathrm{SD}=$ standard deviation; $\mathrm{Sx}=$ surgery; $\mathrm{TB}=$ tooth brush. 
Table 2. Characteristics of the included articles: description of affected sites, implant characteristics, prosthetic factors)

\begin{tabular}{|c|c|c|c|c|c|c|c|c|c|c|c|c|c|c|c|}
\hline \multirow[b]{2}{*}{ Study } & \multirow[b]{2}{*}{ Groups } & \multicolumn{6}{|c|}{ Description of affected sites } & \multicolumn{3}{|c|}{ Implant characteristics } & \multicolumn{4}{|c|}{ Prosthetic factors } & \multirow[b]{2}{*}{$\begin{array}{c}\text { Additional } \\
\text { info }\end{array}$} \\
\hline & & $\begin{array}{l}\text { PDs, } \\
\text { mm }\end{array}$ & $\begin{array}{c}\text { RB loss (SD), } \\
\mathrm{mm}\end{array}$ & ВОР & Exudate & $\underset{\mathrm{mm}}{\mathrm{CAL}(\mathbf{S D}),}$ & ${ }_{\mathrm{mm}}^{\text {Recession (SD), }}$ & Systems & $\begin{array}{c}\text { Time } \\
\text { in function }\end{array}$ & Surface & $\begin{array}{l}\text { Screwed/ } \\
\text { cemented }\end{array}$ & \begin{tabular}{|c|} 
Internal/ \\
external
\end{tabular} & \begin{tabular}{|c|}
$\begin{array}{c}\text { Splinted } \\
\text { non } \\
\text { splinted }\end{array}$ \\
\end{tabular} & $\begin{array}{l}\text { Restoration } \\
\text { type }\end{array}$ & \\
\hline \multirow{2}{*}{ Arisan et al. [20] } & Control & 4 to 6 & $<3 \mathrm{~mm}$ MBL & Yes & $\begin{array}{c}\text { And/or } \\
\text { suppuration }\end{array}$ & NA & NA & \multirow{2}{*}{ Multiple } & 19.4 months & $\begin{array}{l}\text { Rough (acid etching } \\
\text { and sand blasting) }\end{array}$ & Cemented & NA & NA & \multirow{2}{*}{$\begin{array}{l}\text { Fixed metal-ceramic } \\
\text { prostheses }\end{array}$} & \multirow{2}{*}{ Suprastructures removed for measurement. } \\
\hline & Test & 4 to 6 & $<3 \mathrm{~mm} \mathrm{MBL}$ & Yes & $\begin{array}{c}\text { And/or } \\
\text { suppuration }\end{array}$ & NA & NA & & 19.4 months & $\begin{array}{l}\text { Rough (acid etching } \\
\text { and sand blasting) }\end{array}$ & Cemented & NA & NA & & \\
\hline \multirow{2}{*}{ Bassetti et al. [21] } & Control: LDD & 4 to 6 & 0.5 to 2 & \multirow{2}{*}{ Yes } & \multirow{2}{*}{ NA } & $2.72(0.72)$ & $1.68(1.04)$ & \multirow{2}{*}{ Straumann tissue level } & $7.2(2.6-15)$ years & \multirow{2}{*}{ SLA } & \multirow{2}{*}{ Screwed } & \multirow{2}{*}{ NA } & \multirow{2}{*}{ NA } & \multirow{2}{*}{ NA } & \multirow{2}{*}{ NA } \\
\hline & Test: PDT & 4 to 6 & 0.5 to 2 & & & $2.66(0.73)$ & $1.53(0.91)$ & & $7.3(4-14.8)$ years & & & & & & \\
\hline \multirow{2}{*}{ Esposito et al. [22] } & Control & \begin{tabular}{|c} 
Deepest \\
pocket only: \\
6.45
\end{tabular} & Mean $4.73(2.11)$ & \multirow{2}{*}{ Yes } & \multirow{2}{*}{$\begin{array}{l}\text { Pus exudation } \\
\text { and/or soft } \\
\text { tissue swelling } \\
\text { and/or soft } \\
\text { tissue redness }\end{array}$} & \multirow{2}{*}{ NA } & \multirow{2}{*}{ NA } & \multirow{2}{*}{ NA } & 6.13 years & \multirow{2}{*}{ NA } & $\mathrm{NA}$ & $\mathrm{NA}$ & $\mathrm{NA}$ & $\mathrm{NA}$ & In some of the $S x$ treated cases, unsupported \\
\hline & Test & \begin{tabular}{|l} 
Deepest \\
pocket only: \\
6.23
\end{tabular} & Mean $4.4(1.58)$ & & & & & & 5.65 years & & $N A$ & $N A$ & NA & $N A$ & $\begin{array}{l}\text { the clinician's decision } \\
\text { tost }\end{array}$ \\
\hline Hallstrom et al. & Control & $\geq 4$ & $\mid<2 \mathrm{~mm}$ bone loss & Yes & And/or & $\mathrm{NA}$ & $\mathrm{NA}$ & Multiple & 10.9 years & $\mathrm{NA}$ & $\begin{array}{l}\text { Cemented: } 52.6 \% \text {; } \\
\text { Screwed: } 47.4 \%\end{array}$ & NA & & NA & NA \\
\hline [23] & Test & & & & suppuration & NA & NA & Niurupie & 10 years & NA & $\begin{array}{l}\text { Cemented: } 59.1 \% \text {; } \\
\text { Screwed: } 40.9 \%\end{array}$ & NA & $\mathrm{NA}$ & $\mathrm{NA}$ & $\mathrm{NA}$ \\
\hline & $\mathrm{AAD}$ & & & & & $5.2(1.9)$ & $1.5(1.4)$ & & & & & & & & \\
\hline John et al. [24] & $\begin{array}{c}\text { MD (carbon } \\
\text { curettes } \\
+ \text { local antiseptic } \\
\text { therapy) }\end{array}$ & $\geq 4$ & $\begin{array}{c}\text { Loss of } \\
\text { supporting bone } \\
\leq 30 \%\end{array}$ & Yes & Yes & $5(1.5)$ & $1(1.1)$ & Multiple & NA & $\begin{array}{l}\text { Machined surface, } \\
\text { microrough surface }\end{array}$ & Screwed & NA & $\begin{array}{l}\text { angle tour } \\
\text { and } \\
\text { bridgework } \\
\text { restorations }\end{array}$ & NA & Without overhangings or margins \\
\hline Machtei et al. [25] & Control & 6 to 10 & $\geq 2$ & Yes & NA & $7.63(0.3)$ & NA & NA & $\mathrm{NA}$ & $\begin{array}{l}\text { Exclude Titanium Plasma- } \\
\text { sprayed or hydroxylapatite }\end{array}$ & $\mathrm{NA}$ & NA & NA & NA & NA \\
\hline & Test & & & & & $7.88(0.2)$ & & & & coated implants & & & & & \\
\hline & Er:YAG laser & $\geq 5$ & & & & & & & & & & & & & Superstructures were removed to enhance \\
\hline Persson et al. [26] & $\begin{array}{c}\text { AAD subgingival } \\
\text { polishing }\end{array}$ & $\geq 6$ & $\geq 2$ & Yes & suppuration & NA & NA & $\mathrm{NA}$ & NA & $\begin{array}{l}\text { and medium-rough } \\
\text { surfaces }\end{array}$ & $\mathrm{NA}$ & NA & NA & NA & $\begin{array}{l}\text { assessments of } \mathrm{PD} \text { and } \mathrm{BOP} \text { and to improve } \\
\text { the ability to collect bacterial samples. }\end{array}$ \\
\hline Riben-Grundstrom & $\begin{array}{l}\text { Glycine powder } \\
\text { air-polishing }\end{array}$ & & & & And/or & & & & & & & & & & \\
\hline et al. [27] & $\begin{array}{l}\text { Ultrasonic } \\
\text { debridement }\end{array}$ & $\geq 4$ & $\leq 2$ & Yes & suppuration & NA & NA & Multiple & NA & NA & NA & $\mathrm{NA}$ & $\mathrm{NA}$ & $\mathrm{NA}$ & $\mathrm{NA}$ \\
\hline Swierkot et al. [28]. & $\begin{array}{c}\text { Sonic toothbrush } \\
\text { group }\end{array}$ & $\geq 5$ & $<1$ & Yes/ & $\mathrm{NA}$ & 4.64 & 1.24 & Nobel Replace Straight & At least 12 months & Rough & Screwed & $\mathrm{NA}$ & $\mathrm{NA}$ & Single implant or fixed & $\mathrm{NA}$ \\
\hline & $\begin{array}{c}\text { Manual toothbrush } \\
\text { group }\end{array}$ & $\geq 6$ & & no & & 4.41 & 1.28 & Groovy & At least 13 months & & & & & prosthesis & \\
\hline Gomes et al. [29] & \begin{tabular}{|c|} 
Test \\
\end{tabular} & $2.23(0.09)$ & NA & Yes & $\mathrm{NA}$ & $\mathrm{NA}$ & NA & Nobel Biocare & $5.7(2.5)$ years & NA & NA & NA & NA & NA & NA \\
\hline Corbella et al. [30] & Mucositis & NA & NA & \begin{tabular}{|l|} 
Bleeding \\
index $>2$
\end{tabular} & NA & NA & NA & NA & NA & NA & NA & NA & NA & Immediately loaded full- & NA \\
\hline & \begin{tabular}{|c} 
Peri-implantitis \\
Control
\end{tabular} & $>4$ & & & & & & & 80.5 months & & & & & & \\
\hline Costa et al. [31] & Test & $>5$ & No & Yes & Yes & NA & NA & Multiple & 77.4 months & NA & $\mathrm{NA}$ & NA & NA & $\begin{array}{l}\text { Single crowns and// } \\
\text { or fixed partial prosthetic }\end{array}$ & NA \\
\hline & Moderate bone loss & $<5$ & $3.9(0.8)$ & & & $3.8(1.3)$ & $0.5(0.5)$ & & & & & & & & \\
\hline Deppe et al. [32] & \begin{tabular}{|l|} 
Severe bone lost \\
\end{tabular} & 5 to 8 & $6.8(0.8)$ & Yes & $\mathrm{NA}$ & $6.7(0.9)$ & $0.9(1.2)$ & $\mathrm{NA}$ & $\mathrm{NA}$ & $\mathrm{NA}$ & $\mathrm{NA}$ & $\mathrm{NA}$ & $\mathrm{NA}$ & $\mathrm{NA}$ & All restorations were left in situ \\
\hline Schworz et al [23] & $\begin{array}{c}\mathrm{MD}+\text { local } \\
\text { antiseptic } \\
(\mathrm{MD}+\mathrm{CHX})\end{array}$ & $\mathrm{NA}$ & No & Yec & No & $\mathrm{NA}$ & $\mathrm{NA}$ & $\begin{array}{l}\text { Zirconia implants } \\
\text { (ZV4, Zircon Vision }\end{array}$ & $N A$ & $\begin{array}{l}\text { Modified (roughness: } \\
\mathrm{Ra}=\text { approx. } 7 \mu \mathrm{m} / \\
\mathrm{Rz}=\text { approx. } 41 \mu \mathrm{m} \text { ) }\end{array}$ & Screwed & $N$ & $N A$ & $N A$ & $N A$ \\
\hline Schwarz et al. [33] & $\begin{array}{l}\text { Er:YAG laser } \\
\text { therapy }\end{array}$ & $\geq 6$ & $\begin{array}{c}\text { Changes in RB } \\
\text { level }\end{array}$ & Yes & $\begin{array}{l}\text { And/or } \\
\text { suppuration }\end{array}$ & $\mathrm{NA}$ & $\mathrm{NA}$ & $\begin{array}{l}\text { GmbH, Wolfratshausen, } \\
\text { Germany) }\end{array}$ & $\mathrm{NA}$ & $\begin{array}{l}\text { Modified (roughness: } \\
\mathrm{Ra}=\text { approx. } 7 \mu \mathrm{m} / \\
\mathrm{Rz}=\text { approx. } 42 \mu \mathrm{m} \text { ) }\end{array}$ & screwed & NA & $\mathrm{NA}$ & $\mathrm{NA}$ & $N A$ \\
\hline
\end{tabular}

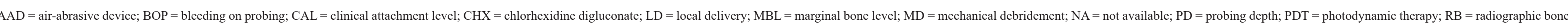
$\mathrm{SD}=$ standard deviation; $\mathrm{SLA}=$ sandblasted and acid-etched implant. 
Table 3. Excluded articles with reasons for exclusion

\begin{tabular}{l|c|c}
\hline \multicolumn{1}{c|}{ Study } & $\begin{array}{c}\text { Year of } \\
\text { publication }\end{array}$ & Reasons for exclusion \\
\hline Mettraux et al. [6] & 2015 & Retrospective study \\
\hline Heitz-Mayfield et al. [10] & 2011 & Short follow-up \\
\hline Ji et al. [11] & 2014 & Short follow-up \\
\hline McKenna et al. [12] & 2013 & Short follow-up \\
\hline Mussano et al. [13] & 2013 & Short follow-up \\
\hline Lerario et al. [14] & 2016 & Retrospective study \\
\hline Parma-Benfenati et al. [15] & 2013 & Review \\
\hline Renvert et al. [16] & 2011 & Same sample as Persson et al. [26] \\
\hline Sahm et al. [17] & 2011 & Same sample as John et al. [24] \\
\hline Schär et al. [18] & 2013 & Same sample as Bassetti et al. [21] \\
\hline Sreenivasan et al. [19] & 2011 & Unclear and incomplete data \\
\hline
\end{tabular}

\section{Treatment interventions of individual studies}

Most of the studies included oral hygiene instructions of using interdental brushes or other required techniques indicated in the protocol before initiating different treatment modalities [20,21, 23-27, $\underline{31-}$ 33]. One study reported self-performed cleaning techniques including certain toothpaste and toothbrush [28]. One article presented the model of systemic administration of antimicrobial agent [23] while Bassetti et al. [21] in 2014 reported the effect of locally delivered antibiotics adjunct to scaling and root planning (SRP) and air-polishing; and another article evaluated the effect of chlorhexidine chip (Perio ${ }^{\circledR}$ chip) in treating peri-implantitis [25]. Of importance to mention is that Bassetti and colleagues [21] repeated the treatment in BOP positive sites after $3,6,9$, and 12 months. Other professional-performed interventions that were presented in the articles include laser, photodynamic therapy, supra-/submucosal mechanical debridement, and air-abrasive devices. Four articles reported the use of lasers in conjunction to SRP $[\underline{20}, \underline{22}, \underline{26}, \underline{33}]$. Lasers employed were diode laser [20], Er:YAG laser $[26, \underline{33}]$ and lightactivated disinfection treatment (FotoSan). Three other articles reported on photodynamic therapy $[\underline{21}, \underline{22}, \underline{32}]$.

\section{Treatment outcomes}

PPD was reported as direct or the percentage change except one article [27]. Majority of studies demonstrated that the change of PPD are within 1 $\mathrm{mm}$. Bleeding index or the percentage of BOP also decreases after different treatment modalities in most of the studies. Regarding the laser studies, 3 studies showed that there were no additional positive effect beyond the traditional mechanical debridement and had limited influence in treating peri-implantitis $[\underline{20}, \underline{22}, 26]$ while other one article presented significant clinical improvement [21]. Regarding selfperformed hygiene techniques, most of the studies demonstrated that with the application of hygiene protocol, it is effective to improve clinical parameters and also keeping the low incidence of developing peri-implant mucositis [28]. For professionalperformed mechanical debridement, studies in general demonstrated effectiveness in reducing inflammation and PPD [24,27,30-32].

\section{Assessment of methodological quality}

The results of risk of bias assessment for included RCTs were summarized in Table 4. In addition, 6 studies were non-RCT and qualitative assessments were analysed with NOS. The mean NOS score for the evaluated studies was $6 \pm 1$.

\section{DISCUSSION}

Dental implants have become the gold standard when aiming at reconstruction of the missing dentition. Decades of investigation have proven dental implants to be reliable alternative providing function and aesthetics with long-term success. However, with the increasing number of fixtures being installed yearly, there has also been a significant increase in the number of patients suffering from peri-implant diseases. According to a recent systematic review peri-implant mucositis and peri-implantitis have a prevalence ranging from 19 to $65 \%$ and from 1 to $47 \%$, respectively. On the other hand, mean prevalence for peri-implant mucosistis and periimplantitis are $43 \%$ and $22 \%$, respectively [34]. Consequently, treatment of peri-implant diseases 
Table 4. Risk of bias assessment of the included studies

\begin{tabular}{l|c|c|c|c|c|c|c}
\hline \multicolumn{1}{c|}{ Study } & $\begin{array}{c}\text { Random } \\
\text { sequence } \\
\text { generation }\end{array}$ & $\begin{array}{c}\text { Allocation } \\
\text { concealment }\end{array}$ & $\begin{array}{c}\text { Blinding of } \\
\text { participants and } \\
\text { personnel }\end{array}$ & $\begin{array}{c}\text { Blinding of } \\
\text { outcome } \\
\text { assessment }\end{array}$ & $\begin{array}{c}\text { Incomplete } \\
\text { outcome } \\
\text { data }\end{array}$ & $\begin{array}{c}\text { Selective } \\
\text { reporting }\end{array}$ & $\begin{array}{c}\text { Other } \\
\text { bias }\end{array}$ \\
\hline Arisan et al. [20] & + & - & - & $?$ & + & + & $?$ \\
\hline Bassetti et al. [21] & + & - & - & + & + & + & $?$ \\
\hline Esposito et al. [22] & + & - & - & + & + & + & + \\
\hline Hallström et al. [23] & + & + & - & - & + & + & + \\
\hline John et al. [24] & + & - & - & + & + & - & $?$ \\
\hline Machtei et al. [25] & + & + & + & + & + & + & $?$ \\
\hline Persson et al. [26] & + & - & - & $?$ & + & + & $?$ \\
\hline Riben-Grundstrom et al. [27] & + & + & - & + & + & - & $?$ \\
\hline Swierkot et al. [28] & + & - & - & + & & + & + \\
\hline
\end{tabular}

$+=$ low risk; $?=$ unclear risk; $-=$ high risk.

has become one of the main focus of the investigations in periodontology. During the last decades, this inflammatory condition has witnessed a tremendous advance in terms of understanding of its aetiology as well as the surgical management. As an example, studies focusing on the different aspects of this disease have been multiplied by more than 100 times during the last two decades.

While initially thought as a periodontitis like lesion surrounding a dental implant, peri-implantitis has recently been related to multiple other variables. In fact, there are a great variety of factors that have been related with marginal bone loss (MBL) and/or periimplantitis that often differ with the ones associated with periodontitis. To name a few: surgical trauma, infection, plaque and poor oral hygiene, alcohol and tobacco consumption, as well as biological bone remodelling [35-38]. Moreover, the possible existence of an imbalance between the implant fixture and the surrounding bone has recently been proposed as a possible etiological factor. Although solely based on narrative reviews [39], the hypothesis of a foreign body reaction as another causative agent for MBL has also been proposed [40]. In addition, the influence of the different implant surfaces and the presence of titanium particles embedded into the surrounding periimplant tissues have been recently investigated.

Regardless of the aetiology of the peri-implant diseases, multiple investigations are being conducted trying to elucidate the most effective treatment approach. However, while the determination of the most effective treatment seems a challenging duty and over the years conflicting results have been shown, the importance of prevention for prompt intervention seems to be in agreement by researchers. Today, the prevention as well as early detection of peri-implant mucositis remains as key components in successful dental implantology. These statements are supported by the effectiveness in treatment of mucositis while treatment of peri-implantitis remains unpredictable. Consequently, current evidence shows that periimplant mucositis can be successfully treated by nonsurgical therapy. Locally delivered antibiotics, lasers, mechanical sub- and supra- gingival SRP, as well as air-polishing, among others can be used for the non-surgical treatment of peri-implant mucositis. In addition, repeated treatment of diseased sites seems to be effective.

Substantial improvements are to be made in research regarding treatment of peri-implantitis and periimplant mucositis. It is of paramount importance the identification of both local and systemic factors affecting the incidence and severity of such conditions for the proper management. Now a day, most of the investigations have failed to provide proper documentation with regard to implant system and position, which have been demonstrated to be significant contributing factors influencing the clinical outcome of different treatment modalities. Moreover, depending on the presence and severity of these local and systemic factors, in many instances, the treatment of choice should be explanation of the fixture.

\section{Limitations}

Within the limitation of the present investigations, the major drawback is the multitude of different definitions regarding peri-implant mucositis and peri-implantitis that were employed in the included investigations. Also, multiple different treatment approaches, different implant designs as well as surface characteristics, and wide variation in terms of follow-up periods may have played a role in the treatment outcomes. 


\section{CONCLUSIONS}

Multitude of different treatment approaches is available for the non-surgical treatment of periimplant diseases. While significant variations exist in term of treatment outcomes, the non-surgical treatment seems to effective for peri-implant mucositis. Self-performed hygiene techniques are effective improving clinical parameters and maintaining a low incidence of developing periimplant mucositis. Professional-performed mechanical debridement is effective in reducing inflammation and pocket depths.

\section{ACKNOWLEDGMENTS AND DISCLOSURE STATEMENTS}

The authors do not have any financial interests, either directly or indirectly, in the products or information listed in the paper. This paper was partially supported by the University of Michigan Periodontal Graduate Student Research Fund.

\section{REFERENCES}

1. Peri-implant mucositis and peri-implantitis: a current understanding of their diagnoses and clinical implications. J Periodontol. 2013 Apr;84(4):436-43. [Medline: 23537178] [doi: 10.1902/jop.2013.134001]

2. Derks J, Schaller D, Håkansson J, Wennström JL, Tomasi C, Berglundh T. Effectiveness of Implant Therapy Analyzed in a Swedish Population: Prevalence of Peri-implantitis. J Dent Res. 2016 Jan;95(1):43-9. [Medline: 26701919] [doi: $10.1177 / 0022034515608832]$

3. Chan HL, Lin GH, Suarez F, MacEachern M, Wang HL. Surgical management of peri-implantitis: a systematic review and meta-analysis of treatment outcomes. J Periodontol. 2014 Aug;85(8):1027-41. [Medline: 24261909] [doi: 10.1902/jop.2013.130563]

4. Renvert S, Roos-Jansåker AM, Claffey N. Non-surgical treatment of peri-implant mucositis and peri-implantitis: a literature review. J Clin Periodontol. 2008 Sep;35(8 Suppl):305-15.[Medline: 18724858] [doi: 10.1111/j.1600-051X.2008.01276.x]

5. Khoshkam V, Chan HL, Lin GH, MacEachern MP, Monje A, Suarez F, Giannobile WV, Wang HL. Reconstructive procedures for treating peri-implantitis: a systematic review. J Dent Res. 2013 Dec;92(12 Suppl):131S-8S. [Medline: 24158331] [PMC free article: 3860060] [doi: 10.1177/0022034513509279]

6. Mettraux GR, Sculean A, Bürgin WB, Salvi GE. Two-year clinical outcomes following non-surgical mechanical therapy of peri-implantitis with adjunctive diode laser application. Clin Oral Implants Res. 2016 Jul;27(7):845-9. [Medline: 26374080]

7. Moher D, Liberati A, Tetzlaff J, Altman DG; PRISMA Group. Preferred reporting items for systematic reviews and metaanalyses: the PRISMA statement. Int J Surg. 2010;8(5):336-41. [Medline: 20171303] [doi: 10.1016/j.ijsu.2010.02.007]

8. Higgins JP, Altman DG, Gøtzsche PC, Jüni P, Moher D, Oxman AD, Savovic J, Schulz KF, Weeks L, Sterne JA; Cochrane Bias Methods Group; Cochrane Statistical Methods Group. The Cochrane Collaboration's tool for assessing risk of bias in randomised trials. BMJ. 2011 Oct 18;343:d5928. [Medline: 22008217] [PMC free article: 3196245] [doi: 10.1136/bmj.d5928]

9. Stang A. Critical evaluation of the Newcastle-Ottawa scale for the assessment of the quality of nonrandomized studies in meta-analyses. Eur J Epidemiol. 2010 Sep;25(9):603-5. [Medline: 20652370] [doi: 10.1007/s10654-010-9491-z]

10. Heitz-Mayfield LJ, Salvi GE, Botticelli D, Mombelli A, Faddy M, Lang NP; Implant Complication Research Group. Anti-infective treatment of peri-implant mucositis: a randomised controlled clinical trial. Clin Oral Implants Res. 2011 Mar;22(3):237-41. [Medline: 21251076] [doi: 10.1111/j.1600-0501.2010.02078.x]

11. Ji YJ, Tang ZH, Wang R, Cao J, Cao CF, Jin LJ. Effect of glycine powder air-polishing as an adjunct in the treatment of peri-implant mucositis: a pilot clinical trial. Clin Oral Implants Res. 2014 Jun;25(6):683-9. [Medline: 23432642] [doi: $10.1111 / \mathrm{clr} .12123$ ]

12. McKenna DF, Borzabadi-Farahani A, Lynch E. The effect of subgingival ozone and/or hydrogen peroxide on the development of peri-implant mucositis: a double-blind randomized controlled trial. Int J Oral Maxillofac Implants. 2013 Nov-Dec;28(6):1483-9. [Medline: 24278915] [doi: 10.11607/jomi.3168]

13. Mussano F, Rovasio S, Schierano G, Baldi I, Carossa S. The effect of glycine-powder airflow and hand instrumentation on peri-implant soft tissues: a split-mouth pilot study. Int J Prosthodont. 2013 Jan-Feb;26(1):42-4. [Medline: 23342332] [doi: $10.11607 /$ ijp.3063]

14. Lerario F, Roncati M, Gariffo A, Attorresi E, Lucchese A, Galanakis A, Palaia G, Romeo U. Non-surgical periodontal treatment of peri-implant diseases with the adjunctive use of diode laser: preliminary clinical study. Lasers Med Sci. 2016 Jan;31(1):1-6. [Medline: 26188854] [doi: 10.1007/s10103-015-1785-7]

15. Parma-Benfenati S, Roncati M, Tinti C. Treatment of peri-implantitis: surgical therapeutic approaches based on peri-implantitis defects. Int J Periodontics Restorative Dent. 2013 Sep-Oct;33(5):627-33. [Medline: 23998151] [doi: $10.11607 / \mathrm{prd} .1549]$ 
16. Renvert S, Lindahl C, Roos Jansåker AM, Persson GR. Treatment of peri-implantitis using an Er:YAG laser or an air-abrasive device: a randomized clinical trial. J Clin Periodontol. 2011 Jan;38(1):65-73. [Medline: 21091527] [doi: 10.1111/j.1600-051X.2010.01646.x]

17. Sahm N, Becker J, Santel T, Schwarz F. Non-surgical treatment of peri-implantitis using an air-abrasive device or mechanical debridement and local application of chlorhexidine: a prospective, randomized, controlled clinical study. J Clin Periodontol. 2011 Sep;38(9):872-8. [Medline: 21770995] [doi: 10.1111/j.1600-051X.2011.01762.x]

18. Schär D, Ramseier CA, Eick S, Arweiler NB, Sculean A, Salvi GE. Anti-infective therapy of peri-implantitis with adjunctive local drug delivery or photodynamic therapy: six-month outcomes of a prospective randomized clinical trial. Clin Oral Implants Res. 2013 Jan;24(1):104-10. [Medline: 22568744] [doi: 10.1111/j.1600-0501.2012.02494.x]

19. Sreenivasan PK, Vered Y, Zini A, Mann J, Kolog H, Steinberg D, Zambon JJ, Haraszthy VI, da Silva MP, De Vizio W. A 6-month study of the effects of $0.3 \%$ triclosan/copolymer dentifrice on dental implants. J Clin Periodontol. 2011 Jan;38(1):33-42. [Medline: 20831669] [doi: 10.1111/j.1600-051X.2010.01617.x ]

20. Arısan V, Karabuda ZC, Arıcı SV, Topçuoğlu N, Külekçi G. A randomized clinical trial of an adjunct diode laser application for the nonsurgical treatment of peri-implantitis. Photomed Laser Surg. 2015 Nov;33(11):547-54. [Medline: 26382562] [doi: 10.1089/pho.2015.3956]

21. Bassetti M, Schär D, Wicki B, Eick S, Ramseier CA, Arweiler NB, Sculean A, Salvi GE. Anti-infective therapy of periimplantitis with adjunctive local drug delivery or photodynamic therapy: 12-month outcomes of a randomized controlled clinical trial. Clin Oral Implants Res. 2014 Mar;25(3):279-87. [Medline: 23560645] [doi: 10.1111/clr.12155]

22. Esposito M, Grusovin MG, De Angelis N, Camurati A, Campailla M, Felice P. The adjunctive use of light-activated disinfection (LAD) with FotoSan is ineffective in the treatment of peri-implantitis: 1-year results from a multicentre pragmatic randomised controlled trial. Eur J Oral Implantol. 2013 Summer;6(2):109-19. [Medline: 23926583]

23. Hallström H, Persson GR, Lindgren S, Olofsson M, Renvert S. Systemic antibiotics and debridement of periimplant mucositis. A randomized clinical trial. J Clin Periodontol. 2012 Jun;39(6):574-81. [Medline: 22571225] [doi: 10.1111/j.1600-051X.2012.01884.x]

24. John G, Sahm N, Becker J, Schwarz F. Nonsurgical treatment of peri-implantitis using an air-abrasive device or mechanical debridement and local application of chlorhexidine. Twelve-month follow-up of a prospective, randomized, controlled clinical study. Clin Oral Investig. 2015 Nov;19(8):1807-14. [Medline: 25605425] [doi: 10.1007/s00784-015-1406-7]

25. Machtei EE, Frankenthal S, Levi G, Elimelech R, Shoshani E, Rosenfeld O, Tagger-Green N, Shlomi B. Treatment of peri-implantitis using multiple applications of chlorhexidine chips: a double-blind, randomized multi-centre clinical trial. J Clin Periodontol. 2012 Dec;39(12):1198-205. [Medline: 23020659] [doi: 10.1111/jepe.12006]

26. Persson GR, Roos-Jansåker AM, Lindahl C, Renvert S. Microbiologic results after non-surgical erbium-doped:yttrium, aluminum, and garnet laser or air-abrasive treatment of peri-implantitis: a randomized clinical trial. J Periodontol. 2011 Sep;82(9):1267-78. [Medline: 21417591] [doi: 10.1902/jop.2011.100660]

27. Riben-Grundstrom C, Norderyd O, André U, Renvert S. Treatment of peri-implant mucositis using a glycine powder air-polishing or ultrasonic device: a randomized clinical trial. J Clin Periodontol. 2015 May;42(5):462-9. [Medline: 25851433] [doi: 10.1111/jcpe.12395]

28. Swierkot K, Brusius M, Leismann D, Nonnenmacher C, Nüsing R, Lubbe D, Schade-Brittinger C, Mengel R. Manual versus sonic-powered toothbrushing for plaque reduction in patients with dental implants: an explanatory randomised controlled trial. Eur J Oral Implantol. 2013 Summer;6(2):133-44. [Medline: 23926585]

29. Gomes SC, Corvello P, Romagna R, Müller LH, Angst PD, Oppermann RV. How do peri-implant mucositis and gingivitis respond to supragingival biofilm control - an intra-individual longitudinal cohort study. Eur J Oral Implantol. 2015 Spring;8(1):65-73. [Medline: 25738180]

30. Corbella S, Del Fabbro M, Taschieri S, De Siena F, Francetti L. Clinical evaluation of an implant maintenance protocol for the prevention of peri-implant diseases in patients treated with immediately loaded full-arch rehabilitations. Int J Dent Hyg. 2011 Aug;9(3):216-22. [Medline: 21356024] [doi: 10.1111/j.1601-5037.2010.00489.x]

31. Costa FO, Takenaka-Martinez S, Cota LO, Ferreira SD, Silva GL, Costa JE. Peri-implant disease in subjects with and without preventive maintenance: a 5-year follow-up. J Clin Periodontol. 2012 Feb;39(2):173-81. [Medline: 22111654] [doi: 10.1111/j.1600-051X.2011.01819.x]

32. Deppe H, Mücke T, Wagenpfeil S, Kesting M, Sculean A. Nonsurgical antimicrobial photodynamic therapy in moderate vs severe peri-implant defects: a clinical pilot study. Quintessence Int. 2013;44(8):609-18. [Medline: 23534047]

33. Schwarz F, John G, Hegewald A, Becker J. Non-surgical treatment of peri-implant mucositis and periimplantitis at zirconia implants: a prospective case series. J Clin Periodontol. 2015 Aug 6. [Medline: 26249545] [doi: 10.1111/jepe.12439]

34. Derks J, Tomasi C. Peri-implant health and disease. A systematic review of current epidemiology. J Clin Periodontol. 2015 Apr;42 Suppl 16:S158-71. [Medline: 25495683] [doi: 10.1111/jcpe.12334]

35. Galindo-Moreno P, Fauri M, Avila-Ortiz G, Fernández-Barbero JE, Cabrera-León A, Sánchez-Fernández E. Influence of alcohol and tobacco habits on peri-implant marginal bone loss: a prospective study. Clin Oral Implants Res. 2005 Oct;16(5):579-86. [Medline: 16164465] [doi: 10.1111/j.1600-0501.2005.01148.x] 
36. Derks J, Håkansson J, Wennström JL, Tomasi C, Larsson M, Berglundh T. Effectiveness of implant therapy analyzed in a Swedish population: early and late implant loss. J Dent Res. 2015 Mar;94(3 Suppl):44S-51S. [Medline: 25503901] [PMC free article: 4541089] [doi: 10.1177/0022034514563077]

37. Daubert DM, Weinstein BF, Bordin S, Leroux BG, Flemming TF. Prevalence and predictive factors for peri-implant disease and implant failure: a cross-sectional analysis. J Periodontol. 2015 Mar;86(3):337-47. [Medline: 25415249] [doi: 10.1902/jop.2014.140438]

38. Suárez-López Del Amo F, Lin GH, Monje A, Galindo-Moreno P, Wang HL. Influence of Soft Tissue Thickness on Peri-Implant Marginal Bone Loss: A Systematic Review and Meta-Analysis. J Periodontol. 2016 Jun;87(6):690-9. [Medline: 26777766] [doi: 10.1902/jop.2016.150571]

39. Trindade R, Albrektsson T, Wennerberg A. Current concepts for the biological basis of dental implants: foreign body equilibrium and osseointegration dynamics. Oral Maxillofac Surg Clin North Am. 2015 May;27(2):175-83. [Medline: 25753575] [doi: 10.1016/j.coms.2015.01.004]

40. Albrektsson T, Dahlin C, Jemt T, Sennerby L, Turri A, Wennerberg A. Is marginal bone loss around oral implants the result of a provoked foreign body reaction? Clin Implant Dent Relat Res. 2014 Apr;16(2):155-65. [Medline: 24004092] [doi: $10.1111 /$ cid.12142]

\section{To cite this article:}

Suárez-López del Amo F, Yu SH, Wang HL.

Non-Surgical Therapy for Peri-Implant Diseases: a Systematic Review

J Oral Maxillofac Res 2016;7(3):e13

URL: http://www.ejomr.org/JOMR/archives/2016/3/e13/v7n3e13.pdf

doi: $10.5037 /$ jomr.2016.7313

Copyright (C) Suárez-López del Amo F, Yu SH, Wang HL. Published in the JOURNAL OF ORAL \& MAXILLOFACIAL RESEARCH (http://www.ejomr.org), 9 September 2016.

This is an open-access article, first published in the JOURNAL OF ORAL \& MAXILLOFACIAL RESEARCH, distributed under the terms of the Creative Commons Attribution-Noncommercial-No Derivative Works 3.0 Unported License, which permits unrestricted non-commercial use, distribution, and reproduction in any medium, provided the original work and is properly cited. The copyright, license information and link to the original publication on (http://www.ejomr.org) must be included. 